\title{
Possibilities of using lighting simulation tools to predict the level of illumination on the workplace from electric lights
}

\author{
Pavel Drabek ${ }^{1, *}$ and Julius Olufemi Ogunleye ${ }^{1}$ \\ ${ }^{1}$ Tomas Bata University in Zlin, Faculty of Applied Informatics, Department of Automation and Control Engineering, Nad Stranemi \\ 4511, 76005 Zlin, Czech Republic
}

\begin{abstract}
Lighting is significant built environment design criteria, and architects or designers always keen to expend efforts to deliver optimal lighting solution. Moreover, the lighting energy consumption represents a significant percentage in a building's energy balance. For these reasons, a suitable way to control the lighting in this field is crucial. The design of control algorithms can be done in various ways, however, in recent years, very useful tools for performing complex lighting assessments are simulation tools. This article deals with a comparison of the results of simulation tools and real measurements and further discusses the possibilities of using these tools to design lighting control algorithms. For the purpose of this paper was used specialized light laboratory where the lighting control is realized via the KNX system. This solution allows the adjustment of luminous intensity and colour temperature for each light separately. Subsequently, the comparison was made for the various configurations of the luminaire settings.
\end{abstract}

\section{Introduction}

Energy use in buildings represents more than one-third of global final energy consumption [1]. Therefore, energy management is a key component of long-term strategies to reduce energy consumption in buildings [2]. From the point of view of individuals, rather than appeal to the investors' sense of society; thus measures to improve energy efficiency must offer investors competitive returns on investment. Although energy conservation can take many forms, the efficient lighting control, in particular, is one of the major trend today [3]. As electric light sources have become more energy efficient and installed lighting power density has declined in recent decades, lighting control has become the primary means to achieve additional energy savings by minimizing or eliminating the use of electric lighting whenever possible [1].

There are many ways how lighting can be controlled. Recently, research in this area has focused on electric lighting control with a minimum number of sensors. The basic input parameters are then the solar parameters, the location of the building, the layout of the interior space and the global value of the solar radiation from one sensor or weather station. Afterwards, computer-based algorithms can control electric lighting and shading devices on daylight apertures to provide appropriate space and task illuminance and to limit daylight or sunlight penetration, reduce window luminance and glare, and maintain acceptable levels of visual control [1]. Many manufacturers have tried to create their own general algorithm, but this approach subsequently exhibits considerable inaccuracies, because the location and geometry of different spaces are not the same.

The design of the control algorithm is then a very complex matter that requires extensive computational assessment and also the real illumination measuring on the workplace because afterwards, the control runs without feedback. Therefore, the effort is to find a certain relation between all the variables involved in the illumination of the spaces. This suggests a relatively time-consuming process, but this process can be greatly speeded up when using certain software tools.

In recent decades, wide varieties of lighting simulation software or tools have been developed to make interior and lighting designs more efficient. At the same time, a large number of studies (or BESTTESTs), for example [4][5][6][7], have been carried out to deal with the comparison of these simulation tools with each other in many aspects. These studies show very similar calculation outputs of illumination of virtual building reference for almost all programs involved. This, however, does not indicate the usability of these tools in real building reference and they are still often used without enough knowledge about their accuracy or limitations.

Based on the above, this paper studies and compares calculation outputs from selected simulation software and values from the real measurement because these types of research are currently very few. For the purpose of this paper, specialized light laboratory at FAI UTB in Zlin was used. This article focuses only on artificial lighting with no daylight involved. This is the first part to be followed by further work in the future.

\footnotetext{
* Corresponding author: pdrabek@utb.cz
} 


\section{Description of the light laboratory}

The light laboratory was built in 2017 and serves for research and development needs at FAI UTB in Zlin. The primary objective of this laboratory was not to specialize in testing high-end luminaries but to address the impact of their effects on indoor environments. The equipment of the laboratory was chosen to examine all types of lighting, natural light, artificial light, and their combination. The laboratory is located on the 3rd floor of the building with windows facing south. It is a room of rectangular shape with dimensions of $8.7 \times 7.3 \times 3.4$ meters. There are work desks that define the work area at a height of $0.8 \mathrm{~m}$ above the floor. The artificial light source consists of luminaires with dimmable electronic ballasts (12 x LED panel CCT 6060 36W, 3600lm) which are also capable of changing the colour of the light $(3000-6500 \mathrm{~K})$. These luminaires are suspended on a HILTI construction that is sliding to change their vertical and horizontal position. In this situation, the lights were placed at a height of $2.5 \mathrm{~m}$ above the floor. To control the penetration rate of daylight, there are shutters connected via blind actor to the KNX. From the inside as secondary dimming elements, there are blinds that are operated manually. This solution allows for complete darkness in the laboratory.

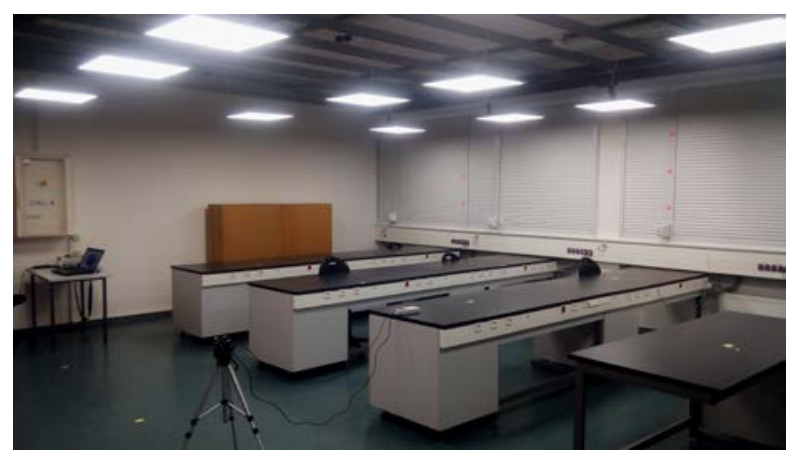

Fig. 1. The light laboratory at FAI UTB in Zlin (photo).

The lighting control is performed by DALI, which is specially designed to control lighting where the KNX DALI-Gateway connects KNX with electronic ballasts equipped with a DALI interface. This interface enables the luminous intensity and colour temperature adjustment for each light separately. The logic itself is based on a control algorithm whose primary objective is to control the inner illumination to a constant level and the goal is to keep the power consumption for lighting at the minimum level and at the same time, meet the hygienic limits, which are given in [8].

\section{Methods}

The measurement and also a comparison was performed only for artificial light with no daylight involved. Evaluation criteria were the horizontal indoor illuminance $\boldsymbol{E}$ [lux] that was collected manually, at specific locations (see Fig. 2), using a measurements set including a datalogger AHLBORN ALMEMO 2390-8 and two light intensity sensors FLA623VL and
FLA613VL, both with the accuracy of 5\% of the measured value. Measurements were collected in 12 observation points, at a height of 0.8 meters above the floor (see Fig. 3).

For the purpose of comparison, the DiaLux Evo software was chosen as the simulation tool. This software was developed by DIAL GmbH DIALux and its a widely used commercial package in lighting design, which is available for free through lighting manufacturers' websites. The exact model of the laboratory was created in this program and the luminaire shape and other luminaire definition in greater detail were downloaded from the manufacturer's catalogue.

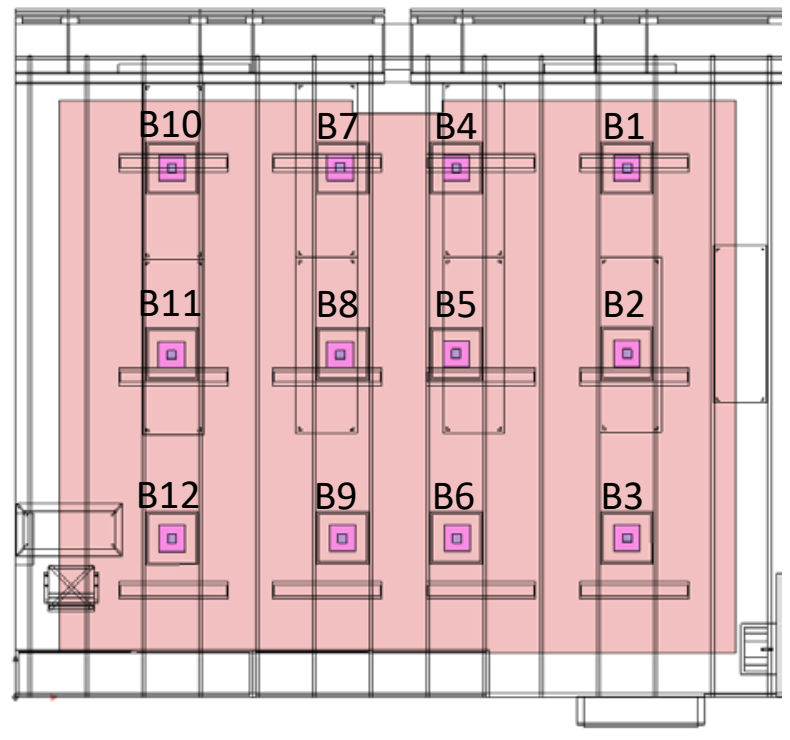

Fig. 2. Model of the light laboratory with the markings of the observed points.

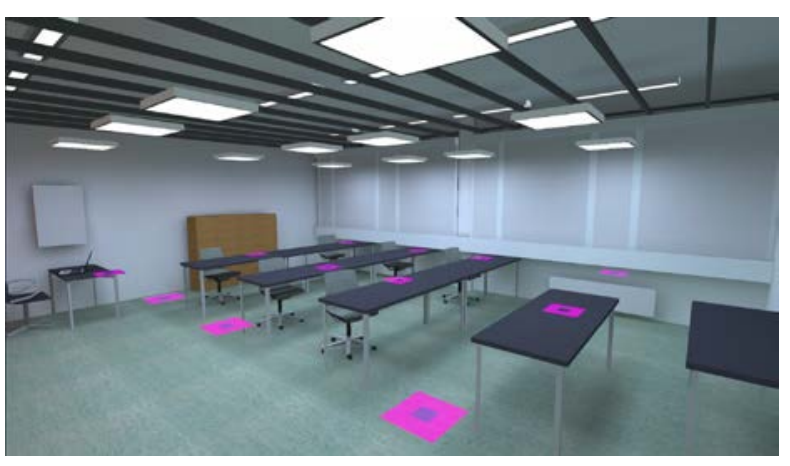

Fig. 3. Model of the light laboratory in $3 \mathrm{D}$ with the observation points.

In the case of real measurement, the procedure proceeded as follows. At first, the workplace illuminance was measured, where all lights were being controlled together. During these measurements, the lighting intensity of all luminaires changed to $14 \%, 53 \%, 72 \%$ and $100 \%$ of their maximum power and this whole process was repeated for different colour temperatures $\boldsymbol{T}_{\mathrm{C}}(3000 \mathrm{~K}, 4000 \mathrm{~K}, 5000 \mathrm{~K}$ and $6000 \mathrm{~K})$.

In contrast to the first phase of measurement, the second one concerned the control of each luminaire separately. The goal was to set the luminaire parameters to achieve uniform illumination levels on a work plane 
throughout the room. Setting the luminous intensity of the lights then depends primarily on their position in the room. Since the luminaires illuminate the space at a certain angle of radiation it is obvious that if the same luminous intensity is set for all luminaires, there will be greater illumination in the middle than in the rest of the room. For this situation, the luminous intensity values were determined experimentally and the required illumination on the work plane was 500 lux.

Afterwards, all the measured data from the building were compared with results from the computer simulation runs in order to determine how close the computer predictions were to the field-monitored values. Description of both phases is provided below in results section.

\section{Results and discussion}

The results presented below are directed towards the analysis of the degree of accuracy of the DIALux Evo software when applied for real buildings. However, in view of the fact that conducted studies have found that calculation outputs from various lighting simulation programs are very identical, we assume that the results from other programs would be similar to those of the DIALux Evo. A great deal of emphasis was put on the correct setting of all the parameters of all elements that affect the resulting illumination value, such as the material used in terms of surface reflectances, the positions of all elements and the parameters of the luminaires.

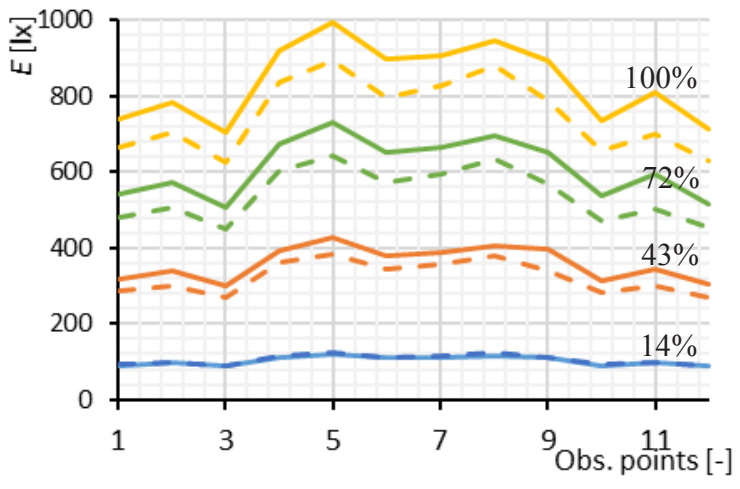

Fig. 4. Illumination values at individual observation points for different luminous intensity, $\boldsymbol{T c}=4000 \mathrm{~K}, \boldsymbol{\Phi}=3600 \mathrm{~lm}$, (continuous line $=$ real measurement, dash line $=$ simulation $)$.

Fig. 4 illustrates the comparison of the results of the workplace illuminance obtained from real measurement and simulation. The comparison is done for four different luminous intensities given in the percentage. As can be seen, the differences in higher luminous intensities are noticeable. In case of luminaires full power, the measured values on average are about 87 lux higher than the results from the simulation. This is mainly due to the fact that in case of dynamic LED CCT type of luminaires, luminous flux $\Phi[\mathrm{lm}]$ can vary widely, depending on the colour temperature [1]. Furthermore, rated luminous flux refers to the initial luminous flux of the new luminaire and also this value should be reached after a certain period of operation [9]; moreover, the value on the luminaire label should indicate the smallest possible value for the entire spectrum of colour temperature but in general, it is usually given for $\boldsymbol{T}_{\mathrm{C}}=4000 \mathrm{~K}$. For this reason, at the beginning of the luminaire operation, we should measure the higher values of the luminous flux than the manufacturer indicates. Therefore, further, it has been tested for which luminous flux value will be obtained similar results to real measurements. The resulting values are $3890 \mathrm{~lm}$ for $3000 \mathrm{~K}, 3920 \mathrm{~lm}$ for $4000 \mathrm{~K}$ (see Fig. 5), $3970 \mathrm{~lm}$ for $5000 \mathrm{~K}$ and almost $4000 \mathrm{~lm}$ for $6000 \mathrm{~K}$. From these values, it can be observed that in the case of these luminaires, with the increasing colour temperature, the luminous flux also increases.

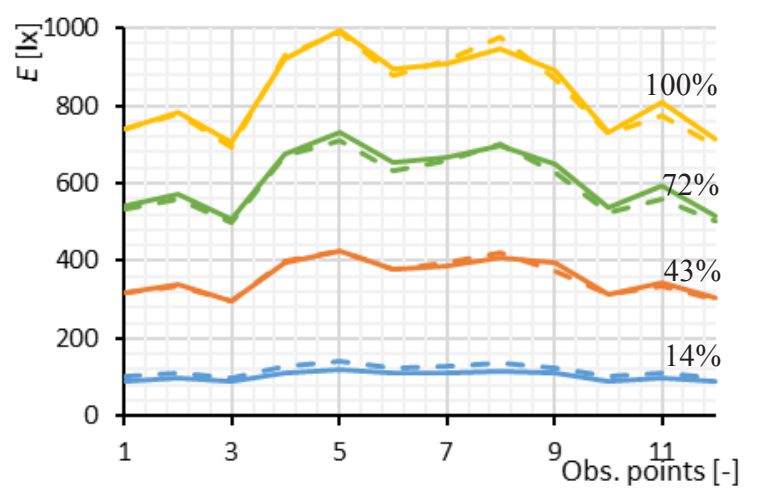

Fig. 5. Illumination values at individual observation points for different luminous intensity, $\boldsymbol{T} \mathrm{c}=4000 \mathrm{~K}, \boldsymbol{\Phi}=39201 \mathrm{~m}$, (continuous line $=$ real measurement, dash line $=$ simulation $)$.

Following are the results of the second measurement phase, concretely lighting control to a constant workplace illumination level of 500 lux. It was desirable to achieve this value only in the task area, it means the place directly below the light. The illumination of the immediate surroundings of the task may be less than the illumination of the task area, more about this is mentioned in [8]. It was therefore desirable to set the luminous intensity of each light so that the illumination levels on a work plane throughout the space was slightly above 500lux. All luminaires were set to colour temperature of $4000 \mathrm{~K}$ and maximum luminous flux of $39201 \mathrm{~m}$ which was then regulated. The resulting comparison is shown in Fig. 6.

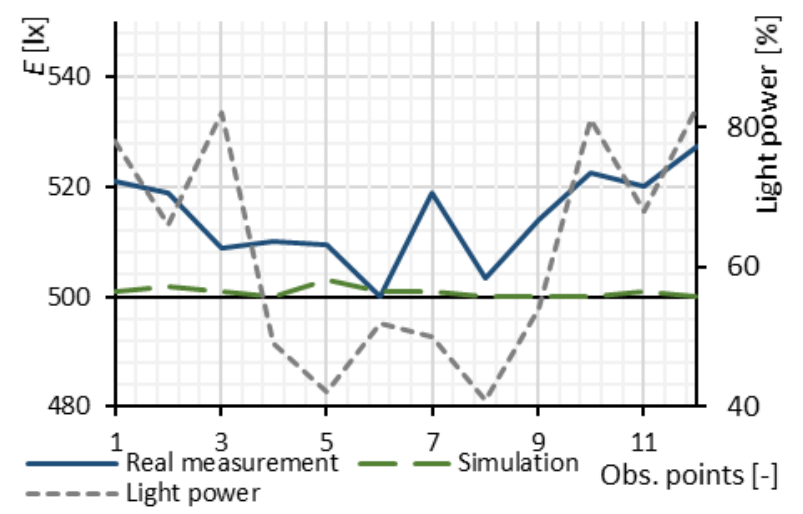

Fig. 6. Lighting control at a constant level of 500 lux and comparison of results. 
As can be seen from Fig. 6, the parameters of luminaires, which in the simulation meant almost constant distribution of the illumination, are in reality slightly different. This can be caused by many factors. One of them could be the accuracy of the electronic ballasts in terms of its regulation, which may not be completely linear. Also, the deviations of the luminous flux and the luminance distribution of each luminaire contribute to the resulting differences. However, these are factors that are directly connected to the light sources and the control system also, and it is very complicated to detect their exact impact. In addition, there are inaccuracies that could have been caused by measuring devices and measurement itself. Even though we are confident with measured data, the manufacturer of the used sensors indicates an inaccuracy of 5\%, which in this case means 25 lux.

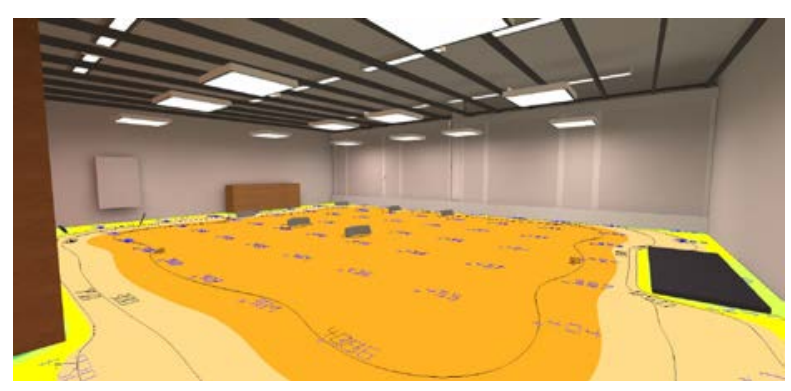

Fig. 7. Example of the illuminance distribution on a work plane throughout the lab.

\section{Conclusion}

Lighting controls are an essential component of any lighting system, serve multiple purposes, and range from simple user-activated switches to advanced scene controllers with variable electric lighting and shading devices adjustment depending on daylight [1][10]. Simulation tools used in light analysis provide accurate results, if the sources involved are perfectly diffuse emitters or if the surfaces involved exhibit perfectly diffuse reflection. However, this is in real building reference very difficult to achieve, hence real values will always show certain inaccuracies.

This paper is a study of the possibilities of using simulation tools to predict the degree of illumination of the indoor environment and it's a first part of the supposed long-term study aimed at defining the accuracy and limitation of the lighting simulation tools. For this purpose, several series of measurements and computer simulations were performed. The software outputs were compared with real building conditions and physical model predictions in an aspect: illumination levels on a work plane throughout the space. These results will be used to create the control algorithm for electric lighting and shutters according to solar information from a weather station. At present, the computational algorithm is not complete, so only the comparison results are provided in this article.

The results of this work show that the basic knowledge of lighting technology and its parameters are necessary for the correct execution of simulations. As can be seen from Fig. 4 and 5, when controlling the illumination, the luminous flux values on a luminaire label must be multiplied by a certain coefficient, which in the case of a new luminaire is less than 1 and its value gradually increases as a function of its operation. With this knowledge, it is possible to use the results from simulation tools to perform the calculation of the light power of individual luminaires, while controlling the illumination to a constant level. Furthermore, in terms of the accuracy of the simulation, the illuminance value calculation for artificial lighting is found within acceptable precisions but opportunity still remains for complex situations with the inclusion of daylight. Therefore, this is what we intend to deal with in the future.

This work was supported by the Ministry of Education, Youth and Sports of the Czech Republic within the National Sustainability Programme project No. LO1303 (MSMT7778/2014) and also by the Internal Grant Agency of Tomas Bata University in Zlin under the project No. IGA/CebiaTech/2018/001.

\section{References}

1. Global Alliance for Building and Construction, The Global Status Report, 2017

2. D. L. DiLaura, K. W. Houser, R. G. Mistrick, G. R. Steffy, The Lighting Handbook - Reference and Application, IES, 10, (2011)

3. OSRAM - Light Management Systems: Energy Savings

4. S. R. Ali, L. Mahjdoubi, A. Khan, Advances in Energy and Power, 3, 91-95 (2015)

5. M. S. Ubbelohde, Ch. Humann, Building and Environment, 1999

6. S. H. Shikder, A. D. Price, M. Mourshed, ESM, 79 (2009)

7. A. D. Galasiu, M. R. Atif, Building and Environment, 37, 372 (1998)

8. EN ISO 12464, Standard, European Committee for Standardization, Brusel (2011)

9. EN ISO 13032, Standard, European Committee for Standardization, Brusel (2017)

10. Lutron, Daylight Sensor - Design and Application Guide, (2014) 